\title{
A pilot study: The association between physical activity level using by accelerometer and postoperative complications after hepatic resection
}

\author{
HIROYA IIDA, TOMOHARU SHIMIZU, HIROMITSU MAEHIRA, NAOMI KITAMURA, \\ HARUKI MORI, TORU MIYAKE, SACHIKO KAIDA and MASAJI TANI \\ Department of Surgery, Shiga University of Medical Science, Otsu, Shiga 520-2192, Japan
}

Received April 25, 2018; Accepted August 17, 2018

DOI: $10.3892 /$ etm.2018.6816

\begin{abstract}
Recently, accelerometers measuring physical activity level have been available to the public. In the present study, it was examined whether the accelerometer could evaluate postoperative outcomes for 12 patients subjected to hepatic resection from August-November 2016. The association was evaluated between the changing pattern of activity level until the postoperative day (POD) 7 and the occurrence of postoperative complications. The median age of patients was 79 years (range, 58-85). Postoperative complications were identified in 6 patients. The activity level in patients with complications was low from POD 1 and was significantly lower than patients without complications following POD 6. The changing pattern of activity level with all included patients could be divided into the following 3 types: Increase type, bell curve type and flat type. Patients without complications exhibited an accelerated increase of postoperative activity level, categorized as increase type. Bell curve type and flat type demonstrated delay of recovery in postoperative activity levels, and were suggested to be associated with the occurrence of postoperative complications. These findings may provide rationale for larger sample studies to evaluate whether
\end{abstract}

Correspondence to: Dr Hiroya Iida, Department of Surgery, Shiga University of Medical Science, Seta Tsukinowa-Cho, Otsu, Shiga 520-2192, Japan

E-mail: hiroya@belle.shiga-med.ac.jp

Abbreviations: ASA classification, American Society of Anesthesiologists Physical Status classification system; ECOG Performance status, Eastern Cooperative Oncology Group Performance status; $\mathrm{BMI}$, body mass index; $\mathrm{HCV}$, positive of hepatitis $\mathrm{C}$ antibody; NBNC, negative of hepatitis $\mathrm{B}$ surface antigen and hepatitis C antibody; AST, aspartate aminotransferase; ALT, alanine aminotransferase; CRP, C-reactive protein; ICGR15, indocyanine green retention rate at $15 \mathrm{~min}$; HCC, hepatocellular carcinoma; PHLF, post-hepatectomy liver failure; SSI, surgical site infection

Key words: accelerometer, physical activity, hepatic resection, hepatectomy, postoperative complication physical activity level measured via accelerometer may be a surrogate marker for postoperative complications.

\section{Introduction}

The population growth in senior citizens is a serious problem worldwide. Accordingly, the increase in prevalence of hepatic resection is associated with the increasing expectation of surgery for elderly patients ( $>65$ years) (1). A number of reports have demonstrated that hepatic resection is acceptable for elderly patients, as the prognosis and complications of elderly patients following hepatic resection are similar to those of young patients (2-5). Conversely, a number of reports have demonstrated that the complications rate of elderly patients was higher than those of younger patients due to elderly patients' fragility (6-8). Therefore, it appeared to be insufficient to evaluate the risk and indication of surgical intervention by only age. Once postoperative complications occur, high medical expenses are required due to prolongation of the hospitalization period and increased use of drugs. Therefore, prevention and prediction of postoperative complications are an important issue for the elderly population receiving surgical treatments. American Society of Anesthesiologists (ASA) classification, Physical Status Classification System and Eastern Cooperative Oncology Group (ECOG) performance status are useful predictors for evaluating the state of activity in patients prior to surgery (9-13). However, both systems include subjective factors scored by estimation; therefore, their accuracy and objectivity are unclear.

The accelerometer activity meter has received attention from the general public since its release in 2010 in developed countries regarding its potential applications in health care and in rehabilitation (14). The accelerometer is a device that contains an acceleration sensor, making it possible to measure calorie consumption while wearing and has been available to the general public in recent years. An accelerometer has a distinct difference from a pedometer in that not only the walking distance but also the exercise intensity such as sitting time, deskwork time, stair climbing time and bicycle riding time are measured. Additionally, modern activity meters can also measure sleeping time and heart rate. 
The association between physical activity and obesity has been evaluated in the field of rehabilitation $(15,16)$. In addition, a number of reports have demonstrated the association between activity level and cardiovascular disease $(17,18)$. Furthermore, physical activity measured by accelerometer was associated with postoperative outcome, such as duration of hospitalization and postoperative complications, in cardiovascular or orthopedic surgery $(19,20)$. However, in abdominal surgery, there has been no report of the association between complications and physical activity via accelerometer.

As a novel predictor of postoperative complications, the present findings suggested that postoperative physical activity level measured by accelerometer is useful for prediction of postoperative complications. In the present study, an accelerometer was used by patients receiving hepatic resection during the postoperative period, and the association between physical activity level and postoperative complications was examined.

\section{Patients and methods}

Patients. Between August and November 2016, 13 patients (age range; 58-85 years; males, 10 and females, 3) received hepatic resection at Shiga University of Medical Science (SUMS) Hospital (Otsu, Japan). All patients with hepatic resection began a postoperative diet from postoperative day (POD) 1 , and if patients were able to eat $>$ half of the diet served in POD 3, infusion via intravenous feeding was ended. If it was less than half amount, infusion was continued. A total of $1 \mathrm{~g}$ cefazolin sodium as an antibiotic was administered only on the day of surgery every $4 \mathrm{~h}$ during the operation, and it was administered every $8 \mathrm{~h}$ following surgery. In cases where infectious complications occurred postoperatively, the antibiotics were re-administered based on the results of various cultures.

Postoperative rehabilitation with the physical therapist in SUMS Hospital was provided every day from POD 1 for all patients who underwent abdominal surgery. A total of 12 patients wore the accelerometer to evaluate postoperative physical activities. One patient was excluded due to complicated sepsis as a result of venous catheter infection and received intensive managements from POD 1.

Accelerometer. Lifecorder ${ }^{\mathrm{TM}}$ (Suzuken Co., Ltd., Nagoya, Japan) was used as the accelerometer to evaluate physical activity. The Lifecorder ${ }^{\mathrm{TM}}$ contains an acceleration sensor and can measure the basal metabolic rate, momentum, number of walks and exercise intensity by inputting the age, height and weight of the patient. This accelerometer consists of an acceleration sensor that detects acceleration, and a computer that calculates basal metabolic rate, momentum and minute momentum. The momentum is calculated by the acceleration index (exercise intensity) divided into 10 levels by the acceleration sensor that recognizes the amplitude and vibration frequency, multiplied by the body weight. The minute momentum is calculated as 0.5 in 10 levels. All measurements are made every $4 \mathrm{sec}$, and the most reliable value is recorded in the device every 2 min. Although division into 10 levels is unique to this model, it has been confirmed that this is correlated with metabolic equivalents (21).
Methods. All enrolled patients wore the Lifecorder ${ }^{\mathrm{TM}} 24 \mathrm{~h}$ daily on the waist from the time of hospitalization until discharge. The activity level was calculated as total amount of momentum and minute momentum per day excluded basal metabolic rate that was expressed as kcal/day. Data from the Lifecorder ${ }^{\mathrm{TM}}$ was analyzed using Lifelyzer 05 Coach $^{\mathrm{TM}}$ analysis software (Suzuken Co., Ltd.).

Patient background, including age, gender, ASA classification (9-12), ECOG performance status (13), body mass index, diabetes mellitus, background liver disease, albumin, alanine aminotransferase, aspartate aminotransferase, bilirubin, platelet count, prothrombin activity, white blood cell count, C-reactive protein and indocyanine green retention rate at $15 \mathrm{~min}$; and operative factors, including primary disease, tumor size, tumor number, operation approach, operation method, number of resection, operation time and bleeding; the degree of physical activity from POD 1 to POD 7; postoperative course and complications were monitored. Postoperative complications were defined according to Clavien-Dindo classification grade II or higher (22). The post-hepatectomy liver failure (PHLF) was defined according to the definition of the International Study Group of Liver Surgery (ISGLS) (23). The grade A of PHLF was defined as no postoperative complication, because it does not affect the postoperative course. The surgical site infection (SSI) was defined by Centre for Disease Control and Prevention guidelines (24).

Statistical analysis. Continuous variables were expressed as the mean \pm standard error of the mean. The degree of physical activity up to the POD 7 was figured and compared for each day with or without complications. Mann-Whitney U analysis was used for the comparison, and $\mathrm{P}<0.05$ was considered to indicate a statistically significant difference. All statistical analyses were performed with SPSS Statistics 24 (IBM Corp., Armonk, NY, USA).

\section{Results}

Patient background. Table I presents patient characteristics, operative factors and postoperative course between patients with and without complications. The median patients' age was 75 and 79 years in the no complication and complication groups, respectively. The primary diseases were 3 patients with hepatocellular carcinoma (HCC) and 3 patients with liver metastasis in the no complication group, and 5 patients with HCC and 1 patient with liver metastasis in the complication group. In the no complication group, there was 1 patient positive for hepatitis $\mathrm{C}(\mathrm{HCV})$; in contrast, there were $5 \mathrm{HCV}$ patients in the complication group. The median albumin levels were 3.8 and $4.1 \mathrm{~g} / \mathrm{dl}$ in no complication and complication patients, respectively. The prothrombin activity level was $102 \%$ in no complication patients and $92 \%$ in complication patients $(\mathrm{P}=0.05)$. The alanine aminotransferase level in no complication patients $(23 \mathrm{U} / \mathrm{l})$ was significantly lower than in complication patients $(52 \mathrm{U} / 1 ; \mathrm{P}=0.04)$. A total of 3 patients were subjected to partial resection and 3 patients were subjected to anatomical resection in no complication patients, and 4 patients were subjected to partial resection and 2 patients were subjected to anatomical resection in complication patients. The ASA classification and ECOG 
Table I. Clinical characteristics of the study patients.

\begin{tabular}{|c|c|c|c|}
\hline Characteristics & Patients without complication $(n=6)$ & Patients with complication $(n=6)$ & P-value \\
\hline Age (years) & $75(58-81)$ & $80(74-85)$ & 0.09 \\
\hline \multicolumn{4}{|l|}{ Gender } \\
\hline Female & $2(33.3)$ & $1(16.7)$ & \multirow[t]{2}{*}{$>0.99$} \\
\hline Male & $4(66.7)$ & $5(83.3)$ & \\
\hline \multicolumn{4}{|l|}{ ASA classification } \\
\hline 1 & $6(100.0)$ & $4(66.7)$ & \multirow[t]{2}{*}{0.45} \\
\hline 2 & $0(0.0)$ & $2(33.3)$ & \\
\hline \multicolumn{4}{|l|}{ ECOG performance status } \\
\hline 0 & $4(66.7)$ & $2(33.3)$ & \multirow[t]{4}{*}{0.74} \\
\hline 1 & $2(33.3)$ & $2(33.3)$ & \\
\hline 2 & $0(0.0)$ & $1(16.7)$ & \\
\hline 3 & $0(0.0)$ & $1(16.7)$ & \\
\hline BMI $\left(\mathrm{kg} / \mathrm{m}^{2}\right)$ & $20.64(17.85-27.39)$ & $20.98(18.03-25.10)$ & $>0.99$ \\
\hline Diabetes mellitus & $2(33.3)$ & $1(16.7)$ & $>0.99$ \\
\hline \multicolumn{4}{|l|}{ Background liver disease } \\
\hline $\mathrm{HCV}$ & $1(16.7)$ & $5(83.3)$ & \multirow[t]{2}{*}{0.08} \\
\hline NBNC & $5(83.3)$ & $1(16.7)$ & \\
\hline Albumin (g/dl) & $3.8(3.4-4.6)$ & $4.1(2.1-4.2)$ & 0.62 \\
\hline ALT (U/l) & $16(9-26)$ & $28(16-83)$ & 0.07 \\
\hline AST (U/l) & $23(17-29)$ & $52(17-79)$ & 0.04 \\
\hline Bilirubin (mg/dl) & $0.6(0.3-1.2)$ & $0.5(0.3-1.1)$ & 0.63 \\
\hline Platelet count $\left(\mathrm{x} 10^{3} / \mu \mathrm{l}\right)$ & $185(110-224)$ & $128(58-298)$ & 0.22 \\
\hline Prothrombin activity $(\%)$ & $102(86-120)$ & $92(64-99)$ & 0.05 \\
\hline White blood count $\left(\times 10^{3} / \mu 1\right)$ & $5.1(4.0-7.7)$ & $4.2(3.3-6.9)$ & 0.19 \\
\hline CRP (mg/dl) & $0.20(0.01-0.82)$ & $0.04(0.03-0.66)$ & 0.63 \\
\hline ICGR15 & $9.4(3.0-21.6)$ & $13.5(4.9-44.0)$ & 0.36 \\
\hline \multicolumn{4}{|l|}{ Primary disease } \\
\hline Liver metastasis & $3(50.0)$ & $1(16.7)$ & \multirow[t]{2}{*}{0.54} \\
\hline $\mathrm{HCC}$ & $3(50.0)$ & $5(83.3)$ & \\
\hline Tumor size $(\mathrm{cm})$ & $2.4(1.0-3.5)$ & $2.7(2.5-5.0)$ & 0.25 \\
\hline \multicolumn{4}{|l|}{ Tumor number } \\
\hline Single & $5(83.3)$ & $4(66.7)$ & \multirow[t]{2}{*}{$>0.99$} \\
\hline Multiple & $1(16.7)$ & $2(33.3)$ & \\
\hline \multicolumn{4}{|l|}{ Operation approach } \\
\hline Open & $2(33.3)$ & $3(50.0)$ & \multirow[t]{2}{*}{$>0.99$} \\
\hline Laparoscopic & $4(66.7)$ & $3(50.0)$ & \\
\hline \multicolumn{4}{|l|}{ Operation method } \\
\hline Partial resection & $3(50.0)$ & $4(66.7)$ & \multirow[t]{2}{*}{$>0.99$} \\
\hline Anatomical resection & $3(50.0)$ & $2(33.3)$ & \\
\hline \multicolumn{4}{|l|}{ Number of resection } \\
\hline Initial resection & $5(83.3)$ & $5(83.3)$ & \multirow[t]{2}{*}{$>0.99$} \\
\hline Repeated resection & $1(16.7)$ & $1(16.7)$ & \\
\hline Operation time (min) & $212(86-313)$ & $241(95-279)$ & $>0.99$ \\
\hline Bleeding (ml) & $10(10-390)$ & $292(10-1,022)$ & 0.06 \\
\hline \multicolumn{4}{|l|}{ PHLF } \\
\hline A & $1(16.7)$ & $0(0.0)$ & \multirow[t]{3}{*}{$>0.99$} \\
\hline B & $0(0.0)$ & $1(16.7)$ & \\
\hline Negative & $5(83.3)$ & $5(83.3)$ & \\
\hline \multicolumn{4}{|l|}{ SSI } \\
\hline Positive (organ/space) & $0(0.0)$ & $1(16.7)$ & $>0.99$ \\
\hline
\end{tabular}


Table I. Continued.

\begin{tabular}{|c|c|c|c|}
\hline Characteristics & Patients without complication $(n=6)$ & Patients with complication $(n=6)$ & P-value \\
\hline Negative & $6(100.0)$ & $5(83.3)$ & \\
\hline \multicolumn{4}{|l|}{ Complication } \\
\hline Pneumonia & 0 & $2(33.3)$ & \\
\hline Refractory pleural effusion & 0 & $1(16.7)$ & \\
\hline Abscess formation & 0 & $1(16.7)$ & \\
\hline Abdominal bleeding & 0 & $1(16.7)$ & \\
\hline Portal thrombus & 0 & $1(16.7)$ & \\
\hline Hospital stay (days) & $8(7-12)$ & $15(12-50)$ & $<0.01$ \\
\hline \multicolumn{4}{|c|}{$\begin{array}{l}\text { Data are presented as n (\%) or median (range). ASA classification, American Society of Anesthesiologists Physical Status classification system; } \\
\text { ECOG performance status, Eastern Cooperative Oncology Group performance status; BMI, body mass index; HCV, positive of hepatitis C } \\
\text { antibody; NBNC, negative of hepatitis B surface antigen and hepatitis C antibody; ALT, aspartate aminotransferase; AST, alanine aminotrans- } \\
\text { ferase; CRP, C-reactive protein; ICGR 15, indocyanine green retention rate at } 15 \text { min; HCC, hepatocellular carcinoma; PHLF, post-hepatectomy } \\
\text { liver failure; SSI, surgical site infection. }\end{array}$} \\
\hline
\end{tabular}

performance status are indicators of general condition. All of the no complication patients and 4 complication patients were categorized as class 1 using the ASA classification, and 2 complication patients were class 2 . A total of 4 and 2 no complication patients were score 0 and 1 in ECOG performance status, respectively. In contrast, complication patients included 1 patient with score 2 and 1 patient with score 3 . However, there was no statistically significant difference between the two groups.

Complications. Complications occurred in 6 patients with refractory pleural effusion, intra-abdominal abscess, intra-abdominal bleeding which required blood transfusion without invasive treatment and portal thrombus each occurring in 1 patient, and 2 patients developing pneumonia. The SSI of organ/space occurred in 1 patient with complication and PHLF occurred in 1 no complication patient with Grade A and 1 complication patient of Grade B. The median postoperative hospital stay was 8 and 15 days in no complication and complication patients, respectively $(\mathrm{P}<0.01)$.

Physical activity level. All 12 patients had their physical activity measured by accelerometer. Fig. 1 presents a transitive graph of activity level in patients with and without complications. Patients with complications exhibited lower physical activities from POD 1 than patients without complications; however, there was no statistically significant difference in the physical activity level between the 2 groups until POD 5 . The physical activity level continuously accelerated in patients without complication following POD 6. Conversely, patients with complication did not exhibit increased physical activity levels following POD 6. Significant differences were observed in physical activity level following POD 6 between patients with and without complications $(\mathrm{P}<0.01)$.

Types of physical activity change. Details of individual activity level are presented in Fig. 2. The alterations in patients' physical activity level were divided into 3 types: Increased type, bell curve type and flat type. The increased type included 6 patients of normal postoperative course and 1 patient with postoperative abdominal bleeding at POD 1 (Fig. 2A). The bell curve type included 2 patients with postoperative complications who had intra-abdominal abscess at POD 5 and refractory pleural effusion at POD 4 (Fig. 2B). The flat type included 2 patients with pneumonia and 1 patient with portal thrombus at POD 5 (Fig. 2C).

\section{Discussion}

Acceleration sensors automatically detect a patient's number of steps, moving distance, burned calories, activity time and sleep time. Certain accelerometers can distinguish the type of exercise (including slow walking, fast walking, running, desk work and bicycle). In the present study, patients without complications demonstrated that the physical activity level gradually increased and quadratic functional increase was observed until discharge, whereas patients with complications demonstrated that the physical activity level was gently decreased in the postoperative course.

The aim of the present study was to evaluate the usefulness of the accelerometer to predict postoperative complications following hepatic resection. Although the present study was preliminary in nature, the change in postoperative physical activity level following POD 6 demonstrated significant difference depending on the presence or absence of complications. The occurrence of postoperative complications observed prior to POD 5 might lead to the decreased physical activity level; therefore, these findings failed to elucidate whether postoperative physical activity level measured by accelerometer might predict postoperative complications. However, 3 representative types of postoperative physical activity changes were proposed by the occurrence of postoperative course recorded by accelerometer as a surrogate marker for postoperative complications.

These 3 types of postoperative physical activity change were as follows: i) increased type, where a continuous elevation of physical activity level which may include patients without complication; ii) bell curve type, where the physical 


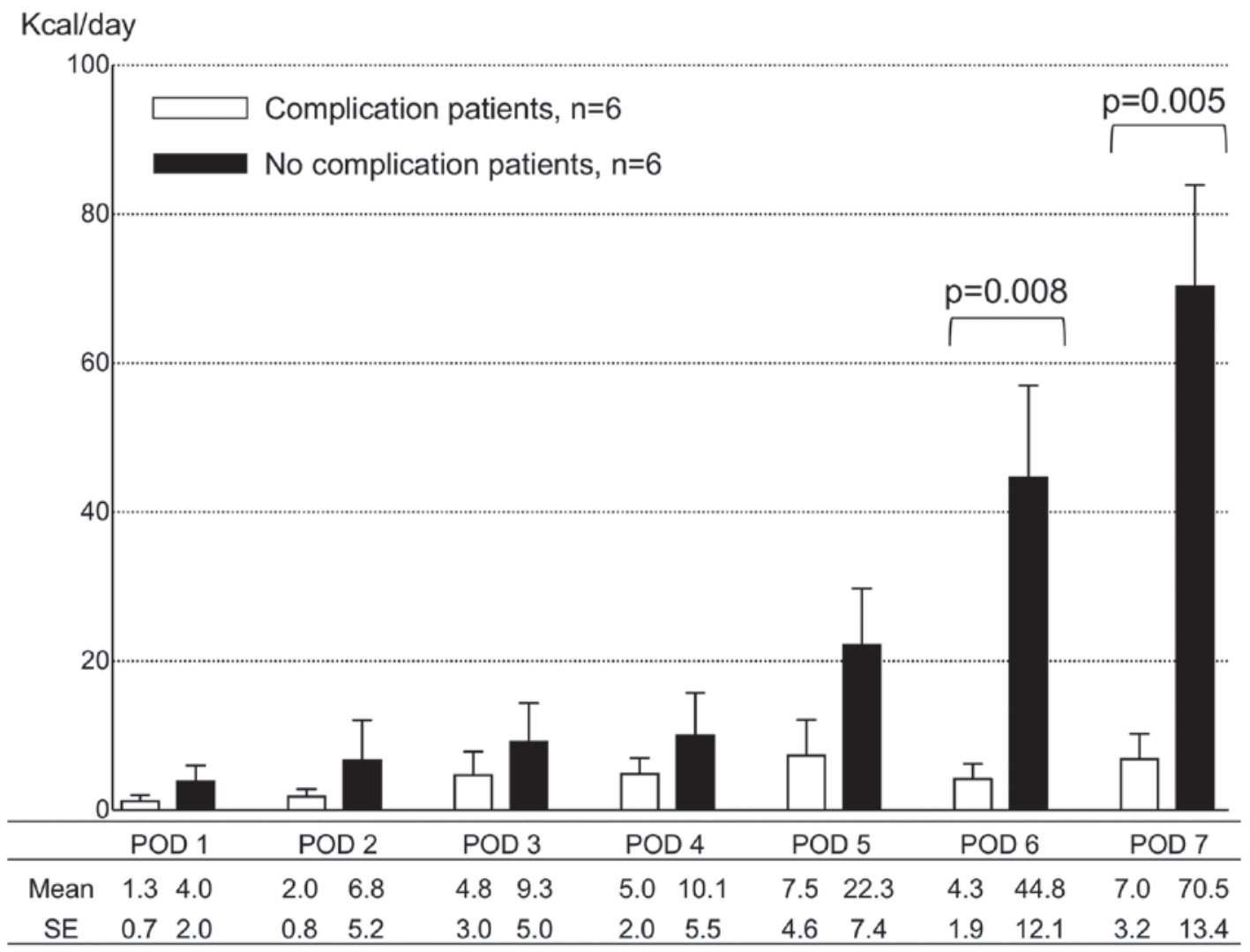

Figure 1. Change of physical activity following operation with or without complications. The vertical axis indicates the degree of physical activity per day and the horizontal axis the number of days following surgery. POD, postoperative day; SE, standard error of the mean.
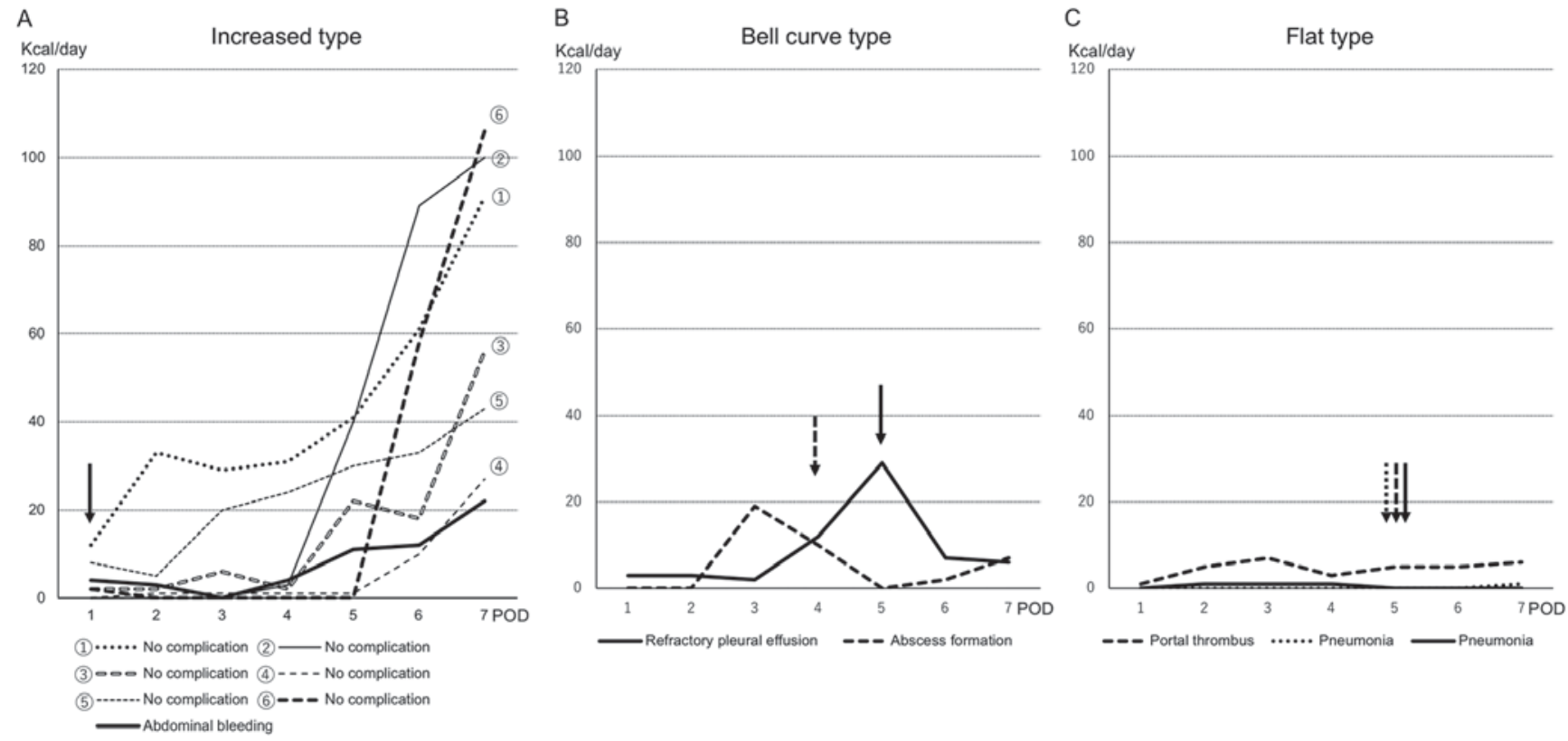

Figure 2. The 3 types of physical activity change following hepatic resection. All included patients were categorized into 1 of the following 3 types: (A) Increased type, (B) bell curve type and (C) flat type. The graphs present all included patients' physical activity changes and postoperative complications. Arrows indicate the day on which the complication occurred. POD, postoperative day,

activity level decreases in the middle period of postoperative course, which may include postoperative complications such as abdominal abscess, bile leak, SSI or effusion. In this type, the physical activities decreased following the occurrence of complications. The occurrence of complications may influence the reduction in physical activities. Therefore, if the amount of physical activity suddenly decreases, complications should be treated as soon as possible and iii) flat type, where low physical activity level continues following surgery. The flat type may include respiratory complications, especially pneumonia. In 
this type, the physical activities are never elevated during the observational period.

Although it is not yet clear due to the small number of patients, the occurrence of pneumonia may be associated with low degree of physical activity in the early postoperative period. Therefore, prevention of pneumonia appeared to require early upregulation of activity level following surgery $(25,26)$.

The limitations of the present study were the small sample size, use of a single center and including some young patients. However, it was demonstrated that the postoperative physical activity level may be a surrogate marker for postoperative complications. This provides rationale for larger sample studies by measuring the postoperative physical activity level. As a future prospect, via a large size study, it may be possible to conduct prediction and prevention of complications depending on the changing pattern of the degree of postoperative physical activity, and it may be possible to determine the schedule of quantity, intensity and time of postoperative rehabilitation to tailor patient management. In addition, it may be possible to demonstrate whether daily and postoperative activity levels lead to differences between young and elderly patients, and whether the influence of the physical activity levels on postoperative complications differs depending on patient's age via larger sample studies.

Furthermore, it is generally considered that a greater amount of daily physical activity reduces the likelihood of postoperative complications. Previously, a number of reports have demonstrated that a few weeks of a cardiopulmonary rehabilitation program prior to surgery reduces postoperative complications (27-29), and a randomized controlled trial is currently ongoing (30). Therefore, it is important to elucidate the association between the degree of daily physical activity prior to operation and postoperative complications. The authors are currently preparing validation of the prediction of complications depending on the changing pattern of postoperative physical activity. In addition, a further study is planned to clarify the association between preoperative physical activity and postoperative complications with a larger number of patients.

In conclusion, the degree of postoperative physical activity measured using an accelerometer may be strongly associated with the postoperative complications following hepatic resection. These findings provide rationale for future studies to evaluate the association between postoperative physical activity levels using an accelerometer and postoperative complications using a larger sample size.

\section{Acknowledgements}

Not applicable.

\section{Funding}

No funding was received.

\section{Availability of data and materials}

The datasets used and/or analyzed during the present study are available from the corresponding author on reasonable request.

\section{Authors' contributions}

HI designed the study and wrote the initial draft of the manuscript. TS and MT contributed to the analysis and interpretation of data, and assisted in the preparation of the manuscript. HiM, NK, HaM, TM and SK contributed to the data collection and interpretation, and critically reviewed the manuscript. The final version of the manuscript was approved by all authors.

\section{Ethics approval and consent to participate}

The present study conformed to the Clinical Research Guidelines and was approved by the Ethics Committee of Shiga University of Medical Science (approval no. 29-191; Otsu, Japan). Written informed consent was obtained from all patients or members of their families prior to surgery.

\section{Patient consent for publication}

Written informed consent was obtained from all patients or members of their families prior to surgery.

\section{Competing interests}

The authors declare that they have no competing interests.

\section{References}

1. Reddy SK, Barbas AS, Turley RS, Gamblin TC, Geller DA, Marsh JW, Tsung A, Clary BM and Lagoo-Deenadayalan S: Major liver resection in elderly patients: A multi-institutional analysis. J Am Coll Surg 212: 787-795, 2011.

2. Poon RT, Fan ST, Lo CM, Liu CL, Ngan H, Ng IO and Wong J: Hepatocellular carcinoma in the elderly: Results of surgical and nonsurgical management. Am J Gastroenterol 94: 2460-2466, 1999.

3. Wu CC, Chen JT, Ho WL, Yeh DC, Tang JS, Liu TJ and P'eng FK: Liver resection for hepatocellular carcinoma in octogenarians. Surgery 125: 332-338, 1999.

4. Shirabe K, Kajiyama K, Harimoto N, Gion T, Tsujita E, Abe T, Wakiyama S, Nagaie T and Maehara Y: Early outcome following hepatic resection in patients older than 80 years of age. World $\mathbf{J}$ Surg 33: 1927-1932, 2009.

5. Kaibori M, Matsui K, Ishizaki M, Saito T, Kitade H, Matsui Y and Kwon AH: Hepatic resection for hepatocellular carcinoma in the elderly. J Surg Oncol 99: 154-160, 2009.

6. Mirici-Cappa F, Gramenzi A, Santi V, Zambruni A, Di Micoli A, Frigerio M, Maraldi F, Di Nolfo MA, Del Poggio P, Benvegnù L, et al: Treatments for hepatocellular carcinoma in elderly patients are as effective as in younger patients: A 20-year multicentre experience. Gut 59: 387-396, 2010.

7. Nanashima A, Abo T, Nonaka T, Fukuoka H, Hidaka S, Takeshita H, Ichikawa T, Sawai T, Yasutake T, Nakao K and Nagayasu T: Prognosis of patients with hepatocellular carcinoma after hepatic resection: Are elderly patients suitable for surgery? J Surg Oncol 104: 284-291, 2011.

8. Nozawa A, Kubo S, Takemura S, Sakata C, Urata Y, Nishioka T, Kinoshita M, Hamano G, Uenishi T and Suehiro S: Hepatic resection for hepatocellular carcinoma in super-elderly patients aged 80 years and older in the first decade of the 21st century. Surg Today 45: 851-857, 2015.

9. Owens WD, Felts JA and Spitznagel EL Jr: ASA physical status classifications: A study of consistency of ratings. Anesthesiology 49: 239-243, 1978.

10. Haynes SR and Lawler PG: An assessment of the consistency of ASA physical status classification allocation. Anaesthesia 50: 195-199, 1995.

11. Ranta S, Hynynen M and Tammisto T: A survey of the ASA physical status classification: Significant variation in allocation among Finnish anaesthesiologists. Acta Anaesthesiol Scand 41: 629-632, 1997. 
12. Mak PH, Campbell RC and Irwin MG; American Society of Anesthesiologists: The ASA physical status classification: Inter-observer consistency. American Society of Anesthesiologists. Anaesth Intensive Care 30: 633-640, 2002.

13. Oken MM, Creech RH, Tormey DC, Horton J, Davis TE, McFadden ET and Carbone PP: Toxicity and response criteria of the Eastern Cooperative Oncology Group. Am J Clin Oncol 5: 649-655, 1982

14. Westerterp KR: Assessment of physical activity: A critical appraisal. Eur J Appl Physiol 105: 823-828, 2009.

15. Bankoski A, Harris TB, McClain JJ, Brychta RJ, Caserotti P, Chen KY, Berrigan D, Troiano RP and Koster A: Sedentary activity associated with metabolic syndrome independent of physical activity. Diabetes Care 34: 497-503, 2011.

16. Colley RC, Garriguet D, Janssen I, Craig CL, Clarke J and Tremblay MS: Physical activity of Canadian children and youth: Accelerometer results from the 2007 to 2009 Canadian Health Measures Survey. Health Rep 22: 15-23, 2011.

17. Healy GN, Matthews CE, Dunstan DW, Winkler EA and Owen N: Sedentary time and cardio-metabolic biomarkers in US adults: NHANES 2003-06. Eur Heart J 32: 590-597, 2011.

18. Stamatakis E, Hamer M, Tilling K and Lawlor DA: Sedentary time in relation to cardio-metabolic risk factors: Differential associations for self-report vs accelerometry in working age adults. Int J Epidemiol 41: 1328-1337, 2012.

19. Cook DJ, Thompson JE, Prinsen SK, Dearani JA and Deschamps C: Functional recovery in the elderly after major surgery: Assessment of mobility recovery using wireless technology. Ann Thorac Surg 96: 1057-1061, 2013.

20. Harding PA, Holland AE, Hinman RS and Delany C: Physical activity perceptions and beliefs following total hip and knee arthroplasty: A qualitative study. Physiother Theory Pract 31: $107-113,2015$

21. Kumahara H, Schutz Y, Ayabe M, Yoshioka M, Yoshitake Y, Shindo M, Ishii K and Tanaka H: The use of uniaxial accelerometry for the assessment of physical-activity-related energy expenditure: A validation study against whole-body indirect calorimetry. Br J Nutr 91: 235-243, 2004.

22. Clavien PA, Barkun J, de Oliveira ML, Vauthey JN, Dindo D, Schulick RD, de Santibañes E, Pekolj J, Slankamenac K, Bassi C, et al: The Clavien-Dindo classification of surgical complications: Five-year experience. Ann Surg 250: 187-196, 2009.
23. Rahbari NN, Garden OJ, Padbury R, Brooke-Smith M, Crawford M, Adam R, Koch M, Makuuchi M, Dematteo RP, Christophi C, et al: Posthepatectomy liver failure: A definition and grading by the International Study Group of Liver Surgery (ISGLS). Surgery 149: 713-724, 2011.

24. Mangram AJ, Horan TC, Pearson ML, Silver LC and Jarvis WR Guideline for prevention of surgical site infection, 1999. Centers for disease control and prevention (CDC) hospital infection control practices advisory committee. Am J Infect Control 27: 97-132; quiz 133-134; discussion 196, 1999.

25. Castelino T, Fiore JF Jr, Niculiseanu P, Landry T, Augustin B and Feldman LS: The effect of early mobilization protocols on postoperative outcomes following abdominal and thoracic surgery: A systematic review. Surgery 159: 991-1003, 2016.

26. Chumillas S, Ponce JL, Delgado F, Viciano V and Mateu M: Prevention of postoperative pulmonary complications through respiratory rehabilitation: A controlled clinical study. Arch Phys Med Rehabil 79: 5-9, 1998.

27. Li C, Carli F, Lee L, Charlebois P, Stein B, Liberman AS, Kaneva P, Augustin B, Wongyingsinn M, Gamsa A, et al: Impact of a trimodal prehabilitation program on functional recovery after colorectal cancer surgery: A pilot study. Surg Endosc 27: 1072-1082, 2013

28. Gillis C, Li C, Lee L, Awasthi R, Augustin B, Gamsa A, Liberman AS, Stein B, Charlebois P, Feldman LS and Carli F: Prehabilitation versus rehabilitation: A randomized control trial in patients undergoing colorectal resection for cancer. Anesthesiology 121: 937-947, 2014.

29. Chan KE, Pathak S, Smart NJ, Batchelor N and Daniels IR: The impact of cardiopulmonary exercise testing on patients over the age of 80 undergoing elective colorectal cancer surgery. Colorectal Dis 18: 578-585, 2016.

30. Onerup A, Angenete E, Bock D, Börjesson M, Fagevik Olsén M, Grybäck Gillheimer E, Skullman S, Thörn SE, Haglind E and Nilsson H: The effect of pre- and post-operative physical activity on recovery after colorectal cancer surgery (PHYSSURG-C): Study protocol for a randomised controlled trial. Trials 18: 212, 2017. 